Herausgeber:

Zentralinstitut für Ernährung der Akademie der Wissenschaften der DDR Direktor: Prof. Dr. habil. H. Hacnel

in Zusammenarbeit mit

der Gesellschaft für Ernührung in der DDR (Vorsitzender: Prof. Dr. habil. H. Schmandke) der Arbeitsgruppe Ernährung beim Nationalen Komitee für Gesundheitserzichung der DDR (Leiter: Prof. Dr. habil. H. - A. Ketz) und dem Warenzeichenverband Diätetieche Erzeugnisso der DDR e.V. (Generaldirektor: F. Schmidt).

Redaktion:

Dr. Friedbert Baum (Chefredakteur), Dr. Jürgen Proll,

Dipl.-Journ. Richard Baier.

Redaktionsbeirat:

Dr. M. Anders, Dr. H. Groß,

Dr. sc. M. Möhr, Dr. R. Schmelter,

Prof. Dr. habil. M. Ulmann, Dr. J. Voigt.

Anschrift der Redaktion:

Zentralinstitut für Eroührung der

Akademie der Wissenschaften der DDR,

DDR-1505 Bergholz-Rehbrücke,

Arthur-Scheunert-Allee 114 - 116.

Verlag:

Akademie-Verlag, DDR-108 Berlin Leipziger Str. 3-4; Fernruf 2236221 oder 2236229

Telex-Nr. 11 4420; Postrcheckkonto:

Berlin: 35021 ; Bank: Staatsbank der DDI, Berlin, Kto,-Nr. : 6836-26-20712.

Veröffentlicht unter der Lizenznummer 1656 des Presseamtes beim Vorsitzenden des Ministerrates der Deutschen Demokratischen Republik.

Gesamtherstellung:

VEB Druckerei „Thomas Müntzer",

DDR-582 Bad Langensalza.

Erscheinungsweise:

Die Zeitschrift "Ernähmungsforschung" erecheint jahrlich in einem Band mit 6 Heften. Bezugspreis je Band 60, - M zuzüglich Versandspesen (Preis für die DDR 30,-M); Preis je Heft 10, -M (Preis für die DDR 5, -M) Bestellnummer dieses Heftes: 1091/23/3.

Alleinige Anzeigenannahme: DEWAG-WERBUNG, DDR-1054 Berlin, Wilhelm-PieckStr. 49 und alle DEWAG-Betriebe in den Bezirksatädten der DDR.

Urheberrecht:

Den Tageszeitungen der Dentschen Demokratischen Republik ist der auszugsweise Nachdruck der Beiträge dieger Zeitechrift bei Quellenangabe honorarfrei gestattet. Ansonsten alle Rechte vorbehalten, insbesondere die der Ubersetzung. Kein Teil dieser Zeitgchrift darf in irgendeiner Form - durch Photokopie, Mikrofilm oder irgend ein anderes Verfahren - ohne schriftliche Genehmigung des Verlages reproduziert werden. (C) 1978 by Akademie-Verlag Berlin. Printed in the German Democratic Republic. AN (EDV) 7721
Qualität im Dienste der Gesundheit . . . . . . . . . . . . . . .

Tendenzen des Lebensmittelverbrauchs und Einflüsse auf die Energieund Nährstoffaufnahme . . . . . . . . . . . . . . . . . . . . 68

Buchbesprechung: Lehrbuch für Köche . . . . . . . . . . . . 72

Buchbesprechung: Früchte der Erde . . . . . . . . . . . . . 72

Zur Ernährungssituation der Landbevölkerung der DDR . . . . . . 73

Begriffe: Limonaden und Brausen mit Frucht-oder Kräutorgoschmack 75

Analyse und Mögliehkeiten der Veränderung der Ernährungssituation der Landbevölkerung . . . . . . . . . . . . . . . . . . . . . 76

Backwaren-Probleme im Bezirk Neubrandenburg . . . . . . . . . 79

Der Wert von Geitreideprodukten für die diätetische Prophylaxe, Behandlung und Metaphylaxe der Fettsucht . . . . . . . . . . . .

Untermediate Moisture Foods - cine neue Klasse von Lobensmittoln ?

Zum Einsatz von Molkenproteinkonzentraten in der Ernährung . . . 86

Karl Lohmann zum 80. Geburtstag . . . . . . . . . . . . . . . 88

Prof. Mašels - 70 Jahre . . . . . . . . . . . . . . . . . 89

Prof. Dr. habil. Max Ulmann zum 80. Geburtstag . . . . . . . . . 89

Adipositas-Behandlung . . . . . . . . . . . . . . . . . . . 89

Mangel und Uberangebot - zwei Erscheinungsformen der Toxizitït von Mineralstoffen . . . . . . . . . . . . . . . . . . . 90

Nahrungsmittel und ihre ernährungsphysiologische Wirkung bei Gustritis und Ulkusleiden . . . . . . . . . . . . . . . . . . . . . . 91

Schulung und Beratung auf dem Gebiet der Gemeinschaftsverpflegung . 93

Zubereitung des 'Tcegetränks . . . . . . . . . . . . . . . . . . 94

Tagungsbericht: XXVIII. Internationaler ärztlicher Fortbildungskurs in Karlovy Vary . . . . . . . . . . . . . . . . . . . . . . . 95

Tagungsbericht: Haltbarkeit von Lebensmitteln . . . . . . . . . 96

\section{Bezugsm oglichkeiten:}

In der DDR über den Postzeitungsvertrieb, über Buchhandlungen oder den Akademie-Verlag Im sozialistischen Ausland über den zuständigen Postzeitungsvertrieb oder eine Buchhandlung fürr fremdsprachige Literatur

In der BRD und Berlin(West) über eine Buchhandlung oder die Auslieferungsstelle KUNST UND WISSEN, Erich Bieber, D-7 Stuttgart 1, Wilhelmstraße 4-6

In Österreich über den Globus-Buchvertrieb,

A 1201 Wien, Höchstădtplatz 3

Im übrigen Ausland über den Internationalen Buch- und Zeitsohriftenhandel; über den Buchexport, Volkseigener AuBenhandelsbetrieb der Deutachen Demokratischen Republik, DDR-701 Leipzig, Postfach 160, oder über den Akademie-Verlag,

DDR-108 Berlin, Leipziger Str. $3-4$

\section{Hinwelse für Autoren}

Die Manuskripte sind in Original-Maschinenschrift einseitig und zweizeilig der Redaktion einzureichen. Pro Zeile 35 oder 70 Anechläge. Der Umfang der Manuskripte sollte mit der Redaktion vereinbart sein. Abbildungen, Tabellen und Zwischentitel sind erwünscht. Es ist zweckmäßig, die Arbeit mit einer kurzen Zusammenfassung oder mit Schlußfolgerungen sowie mit Empfehlungen für die Praxis abzuschließen.

Die Redaktion behält sich eine Uberarbeitung der angenommenen Manuskripte vor; größere Xnderungen nur mit Einverständnis des Autors. Die Beiträge werden honoriert. Der Autor erhalt kostenlos 25 Sonderdrucke seines Beitragee als Fortdrucke. 